\title{
Selecting the "Right" Number of Senses Based on Clustering Criterion Functions
}

\author{
Ted Pedersen and Anagha Kulkarni \\ Department of Computer Science \\ University of Minnesota, Duluth \\ Duluth, MN 55812 USA \\ \{tpederse,kulka020\}@d.umn.edu \\ http://senseclusters.sourceforge.net
}

\begin{abstract}
This paper describes an unsupervised knowledge-lean methodology for automatically determining the number of senses in which an ambiguous word is used in a large corpus. It is based on the use of global criterion functions that assess the quality of a clustering solution.
\end{abstract}

\section{Introduction}

The goal of word sense discrimination is to cluster the occurrences of a word in context based on its underlying meaning. This is often approached as a problem in unsupervised learning, where the only information available is a large corpus of text (e.g., (Pedersen and Bruce, 1997), (Schütze, 1998), (Purandare and Pedersen, 2004)). These methods usually require that the number of clusters to be discovered $(k)$ be specified ahead of time. However, in most realistic settings, the value of $k$ is unknown to the user.

Word sense discrimination seeks to cluster $N$ contexts, each of which contain a particular target word, into $k$ clusters, where we would like the value of $k$ to be automatically selected. Each context consists of approximately a paragraph of surrounding text, where the word to be discriminated (the target word) is found approximately in the middle of the context. We present a methodology that automatically selects an appropriate value for $k$. Our strategy is to perform clustering for successive values of $k$, and evaluate the resulting solutions with a criterion function. We select the value of $k$ that is immediately prior to the point at which clustering does not improve significantly.

Clustering methods are typically either partitional or agglomerative. The main difference is that agglomerative methods start with 1 or $N$ clusters and then iteratively arrive at a pre-specified number $(k)$ of clusters, while partitional methods start by randomly dividing the contexts into $k$ clusters and then iteratively rearranging the members of the $k$ clusters until the selected criterion function is maximized. In this work we have used $\mathrm{K}$ means clustering, which is a partitional method, and the $H 2$ criterion function, which is the ratio of within cluster similarity to between cluster similarity. However, our approach can be used with any clustering algorithm and global criterion function, meaning that the criterion function should arrive at a single value that assesses the quality of the clustering for each value of $k$ under consideration.

\section{Methodology}

In word sense discrimination, the number of contexts $(N)$ to cluster is usually very large, and considering all possible values of $k$ from $1 \ldots N$ would be inefficient. As the value of $k$ increases, the criterion function will reach a plateau, indicating that dividing the contexts into more and more clusters does not improve the quality of the solution. Thus, we identify an upper bound to $k$ that we refer to as deltaK by finding the point at which the criterion function only changes to a small degree as $k$ increases.

According to the $H 2$ criterion function, the higher its ratio of within cluster similarity to between cluster similarity, the better the clustering. A large value indicates that the clusters have high internal similarity, and are clearly separated from each other. Intuitively then, one solution to selecting $k$ might be to examine the trend of $H 2$ scores, and look for the smallest $k$ that results in a nearly maximum $H 2$ value.

However, a graph of $H 2$ values for a clustering 
of the 4 sense verb serve as shown in Figure 1 (top) reveals the difficulties of such an approach. There is a gradual curve in this graph and the maximum value (plateau) is not reached until $k$ values greater than 100 .

We have developed three methods that take as input the $H 2$ values generated from 1 ...deltaK and automatically determine the "right" value of $k$, based on finding when the changes in $\mathrm{H} 2$ as $k$ increases are no longer significant.

\subsection{PK1}

The PK1 measure is based on (Mojena, 1977), which finds clustering solutions for all values of $\mathrm{k}$ from $1 . . N$, and then determines the mean and standard deviation of the criterion function. Then, a score is computed for each value of $k$ by subtracting the mean from the criterion function, and dividing by the standard deviation. We adapt this technique by using the $H 2$ criterion function, and limit $k$ from 1 ...delta $K$ :

$$
P K 1(k)=\frac{H 2(k)-\operatorname{mean}(H 2[1 \ldots \text { deltaK }])}{\operatorname{std}(H 2[1 \ldots \text { deltaK }])}
$$

To select a value of $k$, a threshold must be set. Then, as soon as $P K 1(k)$ exceeds this threshold, $k-1$ is selected as the appropriate number of clusters. We have considered setting this threshold using the normal distribution based on interpreting $P K 1$ as a $\mathrm{z}$-score, although Mojena makes it clear that he views this method as an "operational rule" that is not based on any distributional assumptions. He suggests values of 2.75 to 3.50 , but also states they would need to be adjusted for different data sets. We have arrived at an empirically determined value of -0.70 , which coincides with the point in the standard normal distribution where $75 \%$ of the probability mass is associated with values greater than this.

We observe that the distribution of $P K 1$ scores tends to change with different data sets, making it hard to apply a single threshold. The graph of the $P K 1$ scores shown in Figure 1 illustrates the difficulty - the slope of these scores is nearly linear, and as such the threshold (as shown by the horizontal line) is a somewhat arbitrary cutoff.

\subsection{PK2}

$P K 2$ is similar to (Hartigan, 1975), in that both take the ratio of a criterion function at $k$ and $k-1$,
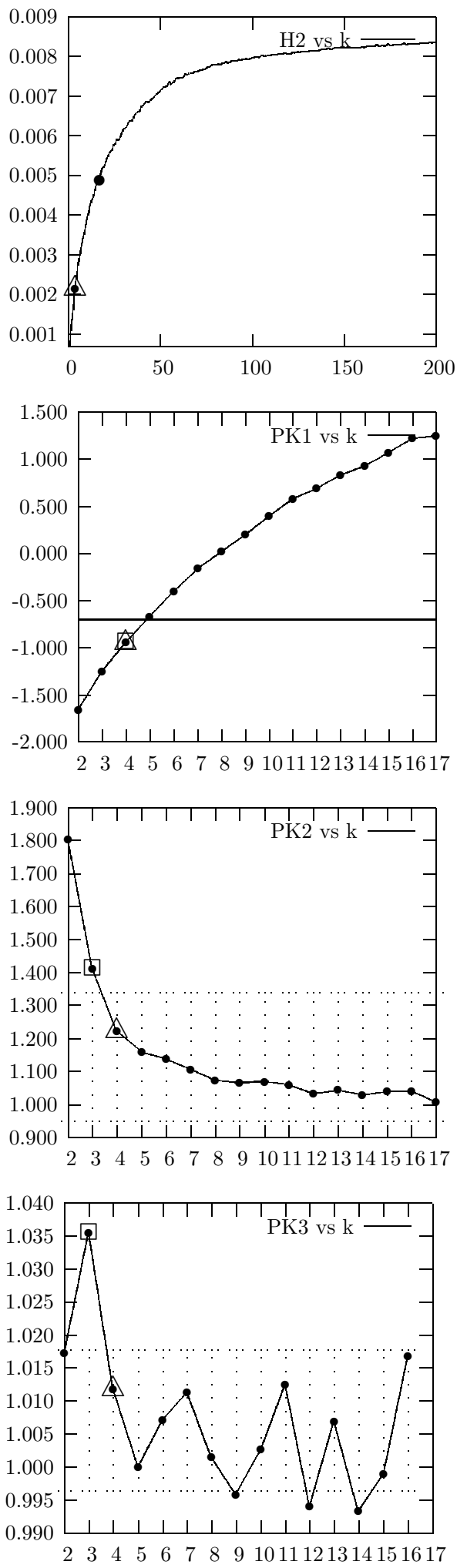

Figure 1: Graphs of $\mathrm{H} 2$ (top) and PK 1-3 for serve: Actual number of senses (4) shown as triangle (all), predicted number as square (PK1-3), and deltaK (17) shown as dot (H2) and upper limit of $k$ (PK1-3). 
in order to assess the relative improvement when increasing the number of clusters.

$$
P K 2(k)=\frac{H 2(k)}{H 2(k-1)}
$$

When this ratio approaches 1 , the clustering has reached a plateau, and increasing $k$ will have no benefit. If $P K 2$ is greater than 1 , then an additional cluster improves the solution and we should increase $k$. We compute the standard deviation of $P K 2$ and use that to establish a boundary as to what it means to be "close enough" to 1 to consider that we have reached a plateau. Thus, $P K 2$ will select $k$ where $P K 2(k)$ is the closest to (but not less than) $1+$ standard deviation(PK2[1...deltaK]).

The graph of $P K 2$ in Figure 1 shows an elbow that is near the actual number of senses. The critical region defined by the standard deviation is shaded, and note that $P K 2$ selected the value of $k$ that was outside of (but closest to) that region. This is interpreted as being the last value of $k$ that resulted in a significant improvement in clustering quality. Note that here $P K 2$ predicts 3 senses (square) while in fact there are 4 actual senses (triangle). It is significant that the graph of $P K 2$ provides a clearer representation of the plateau than does that of $H 2$.

\subsection{PK3}

$P K 3$ utilizes three $k$ values, in an attempt to find a point at which the criterion function increases and then suddenly decreases. Thus, for a given value of $k$ we compare its criterion function to the preceding and following value of $k$ :

$$
P K 3(k)=\frac{2 \times H 2(k)}{H 2(k-1)+H 2(k+1)}
$$

$P K 3$ is close to 1 if the three $H 2$ values form a line, meaning that they are either ascending, or they are on the plateau. However, our use of deltaK eliminates the plateau, so in our case values of 1 show that $k$ is resulting in consistent improvements to clustering quality, and that we should continue. When $P K 3$ rises significantly above 1 , we know that $k+1$ is not climbing as quickly, and we have reached a point where additional clustering may not be helpful. To select $k$ we chose the largest value of $P K 3(k)$ that is closest to (but still greater than) the critical region defined by the standard deviation of $P K 3$. This is the last point where a significant increase in $H 2$ was observed.
Note that the graph of $P K 3$ in Figure 1 shows the value of $P K 3$ rising and falling dramatically in the critical region, suggesting a need for additional points to make it less localized.

PK3 is similar in spirit to (Salvador and Chan, 2004), which introduces the $\mathrm{L}$ measure. This tries to find the point of maximum curvature in the criterion function graph, by fitting a pair of lines to the curve (where the intersection of these lines represents the selected $k$ ).

\section{Experimental Results}

We conducted experiments with words that have 2 , 3,4 , and 6 actual senses. We used three words that had been manually sense tagged, including the 3 sense adjective hard, the 4 sense verb serve, and the 6 sense noun line. We also created 19 name conflations where sets of 2, 3, 4, and 6 names of persons, places, or organizations that are included in the English GigaWord corpus (and that are typically unambiguous) are replaced with a single name to create pseudo or false ambiguities. For example, we replaced all mentions of Bill Clinton and Tony Blair with a single name that can refer to either of them. In general the names we used in these sets are fairly well known and occur hundreds or even thousands of times.

We clustered each word or name using four different configurations of our clustering approach, in order to determine how consistent the selected value of $k$ is in the face of changing feature sets and context representations. The four configurations are first order feature vectors made up of unigrams that occurred 5 or more times, with and without singular value decomposition, and then second order feature vectors based on bigrams that occurred 5 or more times and had a log-likelihood score of 3.841 or greater, with and without singular value decomposition. Details on these approaches can be found in (Purandare and Pedersen, 2004).

Thus, in total there are 22 words to be discriminated, 7 with 2 senses, 6 words with 3 senses, 6 with 4 senses, and 3 words with 6 senses. Four different configurations of clustering are run for each word, leading to a total of 88 experiments. The results are shown in Tables 1,2 , and 3. In these tables, the actual numbers of senses are in the columns, and the predicted number of senses are in the rows.

We see that the predicted value of $P K 1$ agreed 
Table 1: k Predicted by PK1 vs Actual k

\begin{tabular}{c|rrrr|r}
\cline { 2 - 5 } \multicolumn{1}{c|}{} & 2 & 3 & 4 & 6 & \\
\hline 1 & 6 & 6 & 3 & 3 & 18 \\
2 & $\mathbf{5}$ & 5 & 1 & 3 & 14 \\
3 & 4 & $\mathbf{1}$ & 7 & 2 & 14 \\
4 & 6 & 5 & $\mathbf{7}$ & 1 & 19 \\
5 & 4 & 2 & 1 & & 7 \\
6 & 2 & 3 & 3 & $\mathbf{2}$ & 10 \\
7 & 1 & 1 & & & 2 \\
8 & & & 1 & & 1 \\
9 & & 1 & 1 & & 2 \\
11 & & & & 1 & 1 \\
\hline & 28 & 24 & 24 & 12 & 88
\end{tabular}

Table 2: k Predicted by PK2 vs Actual k

\begin{tabular}{c|cccc|r}
\cline { 2 - 5 } \multicolumn{1}{c|}{} & 2 & 3 & 4 & 6 & \\
\hline 1 & & 3 & & 1 & 4 \\
2 & $\mathbf{8}$ & 5 & 7 & 6 & 26 \\
3 & 8 & $\mathbf{1 0}$ & 8 & 2 & 30 \\
4 & 4 & 2 & $\mathbf{3}$ & & 9 \\
5 & 1 & & 3 & 2 & 6 \\
6 & 1 & 2 & & $\mathbf{1}$ & 4 \\
7 & 2 & & & & 2 \\
9 & 1 & 1 & & & 2 \\
10 & & 1 & 2 & & 3 \\
11 & 1 & & & & 1 \\
12 & & & 1 & & 1 \\
17 & 2 & & & & 2 \\
\hline & 28 & 24 & 24 & 12 & 88
\end{tabular}

with the actual value in 15 cases, whereas $P K 3$ agreed in 17 cases, and $P K 2$ agreed in 22 cases. We observe that $P K 1$ and $P K 3$ also experienced considerable confusion, in that their predictions were in many cases several clusters off of the correct value. While $P K 2$ made various mistakes, it was generally closer to the correct values, and had fewer spurious responses (very large or very small predictions). We note that the distribution of $P K 2$ 's predictions were most like those of the actual senses.

\section{Conclusions}

This paper shows how to use clustering criterion functions as a means of automatically selecting the number of senses $k$ in an ambiguous word. We have found that $P K 2$, a ratio of the criterion functions for the current and previous value of $k$, is
Table 3: k Predicted by PK3 vs Actual k

\begin{tabular}{c|cccc|r}
\cline { 2 - 5 } \multicolumn{1}{c|}{} & 2 & 3 & 4 & 6 & \\
\hline 1 & 3 & 4 & 1 & 1 & 9 \\
2 & $\mathbf{1 3}$ & 9 & 12 & 4 & 38 \\
3 & 4 & $\mathbf{3}$ & 4 & 4 & 15 \\
4 & 2 & 2 & $\mathbf{1}$ & 1 & 6 \\
5 & 2 & 1 & 1 & 1 & 5 \\
6 & 1 & 2 & 3 & & 6 \\
7 & 1 & 1 & 1 & & 3 \\
9 & & & & 1 & 1 \\
10 & 1 & & & & 1 \\
11 & & 2 & & & 2 \\
12 & 1 & & & & 1 \\
13 & & & 1 & & 1 \\
\hline & 28 & 24 & 24 & 12 & 88
\end{tabular}

most effective, although there are many opportunities for future improvements to these techniques.

\section{Acknowledgments}

This research is supported by a National Science Foundation Faculty Early CAREER Development Award (\#0092784). All of the experiments in this paper were carried out with the SenseClusters package, which is freely available from the URL on the title page.

\section{References}

J. Hartigan. 1975. Clustering Algorithms. Wiley, New York.

R. Mojena. 1977. Hierarchical grouping methods and stopping rules: An evaluation. The Computer Journal, 20(4):359-363.

T. Pedersen and R. Bruce. 1997. Distinguishing word senses in untagged text. In Proceedings of the Second Conference on Empirical Methods in Natural Language Processing, pages 197-207, Providence, RI, August.

A. Purandare and T. Pedersen. 2004. Word sense discrimination by clustering contexts in vector and similarity spaces. In Proceedings of the Conference on Computational Natural Language Learning, pages 41-48, Boston, MA.

S. Salvador and P. Chan. 2004. Determining the number of clusters/segments in hierarchical clustering/segmentation algorithms. In Proceedings of the 16th IEEE International Conference on Tools with AI, pages 576-584.

H. Schütze. 1998. Automatic word sense discrimination. Computational Linguistics, 24(1):97-123. 